\title{
Voyager's final Solar System rendezvous
}

\section{- High clouds and hurricanes on Neptune - Triton marked by glaciers and ice volcanoes}

\section{Pasadena, California}

As Voyager 2 rounded Neptune $5,000 \mathrm{~km}$ above the clouds and sailed within 25,000 $\mathrm{km}$ of the large moon Triton, it ended its spectacular 12-year mission with a flourish.

Neptune, the last of the giant gaseous planets in the Solar System, turned out to be a more visually appealing object than the generally featureless blue globe of Uranus. On Neptune, Voyager saw high cirrus clouds and a number of large visible features, some bright and some dark, that maintained their identity over the duration of close approach. And Voyager's close pass by Triton revealed a small world marked by recent and probably continuing activity: despite the intense cold, which makes water ice on Triton as unyielding as rock on Earth, Neptune's biggest moon is covered by flows, frozen lakes and 'icy volcanoes'.

Voyager 2, like Voyager 1, will now coast on towards interstellar space, and should remain in radio contact for another thirty years. It will send back measurements of the intensity of the solar wind, and may eventually cross the heliopause, where true interstellar space takes over from the region dominated by the influence of the Sun, but there is nothing more to take pictures of. At Voyager mission control, the Jet Propulsion Laboratory (JPL) in Pasadena, California, VicePresident Dan Quayle came to give the spacecraft a send-off, and as the stream of data from the encounter dwindles, the specialist teams will return to their home institutions to puzzle over their new discoveries. JPL is now turning its attention to the future (see page 669).

The atmospheric activity found on Neptune came as a surprise. Uranus, about the same size as Neptune and similarly shrouded in a dense methane atmosphere, presented a bland face to Voyager three and half years ago, and there was reason to think that Neptune, half as far again from the Sun and correspondingly colder, would be equally inactive.

But even a few months before the encounter, images from Voyager began to reveal large and conspicuous markings on Neptune. The most prominent became known as the Great Dark Spot, in recognition of its superficial similarity to Jupiter's Great Red Spot, and as Voyager neared its destination, a handful of other features appeared. A smaller dark spot moves around the planet close to the south pole, and as it grew in size on successive images, a bright spot appeared in its centre. Between the Great Dark Spot and the smaller one is the 'scooter', a small bright feature that moved around Neptune once every 16 hours, rapidly overtaking the dark spots, whose rotation period is closer to 17 hours.

Scattered across Neptune in a number of latitude bands are bright streaks that were instantly and fittingly described as cirrus clouds. Although a handful of cirrus features appear around the Great Dark Spot in fairly fixed positions, most of the high bright clouds changed from one rotation to another. This gave atmospheric dynamicists a problem: to estimate the circulation speed of the atmosphere, they need to see features that can be
Navigating to Neptune

ON 21 August, 4 days and 6 million km before closest approach, Voyager 2 made its final course adjustment, a sideways nudge in velocity of half a metre per second in an overall speed of $20 \mathrm{~km}$ per second. This delicate change, shifting Voyager's arrival point at Triton by $200 \mathrm{~km}$, put it drectly behind the moon with respect both to the Earth and the Sun so that two occultations could be observed.

The apparently easy success of this finetuning of the trajectory conceals 12 years' worth of practised ingenuity on the part of the JPL scientists and technicians. Voyager's main thrusters, normally used for steering, cannot be fired during a planetary encounter because they heat up the radio receiver and, because of a long-standing malfunction, would cause a loss of contact with Earth for two or three days until the electronics cool down again.

During a planetary encounter, Voyager is steered instead with its attitude adjustment thrusters, which normally fire automatically every 20 minutes or so to keep the radio antenna pointed to Earth. Firing one of these sets Voyager spinning about the axis of the radio dish; when half a turn has been completed, another thruster can be fired to set the spacecraft rotating back the same way. Finally, the first thruster is fired again to stop rotation. The rocket firings are all in the same direction, perpendicular to the direction of motion; the net effect is to edge Voyager crablike across the sky, without loss of radio contact.

Only after this manoeuvre, when Voyager's passage through the neptunian system was calculated to an accuracy of $\mathbf{3 0}$ $\mathrm{km}$, was the final set of instructions for the encounter prepared and transmitted. Some retargeting of the cameras was done to get close-up shots of two of the newly discovered moons, N1 and N2, and of segments of the rings.

On Wednesday afternoon, 23 August, Voyager's instructions for the encounter were transmitted. Because of the eighthour interval between sending signals and receiving confirmation of their arrival, the command sequence was sent six times to ensure error-free receipt. Voyager's memory, state-of-the-art 12 years ago, can hold only 2,000 words of instructions: during the encounter, when the detectors must be turned constantly from one target to another, the meagre space is barely enough to hold two days' worth of instructions.

The finishing touch was delivered on Thursday morning, about $\mathbf{1 2}$ hours before closest approach. From final course determinations, an exact set of timings for the encounter was calculated and transmitted, telling Voyager when to execute the already installed series of commands. From this time until late Saturday afternoon, when it would be $2,500,000 \mathrm{~km}$ beyond Neptune, Voyager was on its own. 


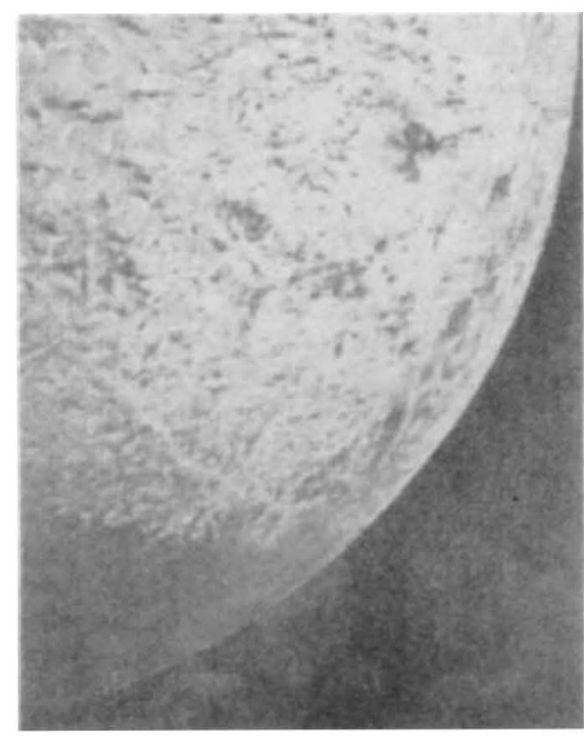

Triton: there's no such thing as a 'dead world'. unambiguously identified over several rotation periods. Around the Great Red Spot of Jupiter, for example, a multitude of recognizable smaller clouds pass by, permitting the rotation sense of the spot to be determined. But around Neptune's Great Dark Spot, the pattern of cirrus clouds is different on every image. Several days after the encounter, it was still impossible to decide if the Great Dark Spot is a cyclone or an anticyclone; as on Earth, the sense of rotation is different according to whether the spot is a low- or high-pressure region.

On some remarkable pictures, cirrus clouds are seen to cast shadows on the basal cloud layer; the height of the cirrus layer was estimated at about $50 \mathrm{~km}$. The dark spots appear to be about halfway between the high and low cloud layers.

The high clouds are methane condensations; the lower clouds are thought to consist of hydrogen sulphide. Sunlight acting on methane in the upper atmosphere probably creates heavier hydrocarbons, which freeze and sink; lower in the atmosphere, where the temperature rises again, they evaporate and decompose back to methane, which rises in plumes to form the visible clouds. On Neptune, further from the Sun than Uranus, most of the energy available to drive atmospheric dynamics is internal heat, welling up from below, rather than solar energy impinging on the upper atmosphere.

Passing beyond Neptune, Voyager 2 saved some of the most startling images of its career for last. Triton, a cold and presumably icy place $2,700 \mathrm{~km}$ in diameter, was far from dead. At a quick glance, cratering on Triton seems not to be heavy, implying that the surface has been processed somehow in fairly recent times the past 100 million years, for example. There had been suggestions that Triton would look 'new'; it has a uniquely odd orbit, circular but inclined to Neptune's axis, and retrograde. This makes it almost certainly a captured body, and suggests that in earlier times its orbit was probably not circular. As tidal forces circularized Triton's orbit, the strains on the moon's body would have heated it up, perhaps enough to melt water ice.

This indeed seems to have been the case. Parts of Triton's surface seem to have 'frozen lakes', resembling lunar maria. In places, the frozen lakes are stepped, suggesting a succession of meltings and re-freezings. But there is much more. Triton's inclined orbit, coupled with Neptune's, means that over a seasonal cycle of about a thousand years the Sun can be overhead anywhere from 50 degrees south to 50 degrees north. At the moment of Voyager's fly-by, the Sun was far to the south as seen from Triton, and near its south pole the surface markings were reminiscent of a martian summer, when the Sun is warm enough to evaporate the frozen carbon dioxide polar caps. On Triton, the ices are methane and nitrogen, but the process seems to be the same. Methane ice on Triton lends it a pinkish colour, darkly mottled by radiation damage from high-energy ionized particles which stream onto the the moon's surface and create darker hydrocarbons from the methane.

Elsewhere there is evidence of smaller flows that have filled in valleys and fissures. These are probably glaciers of methane and nitrogen which, like ice glaciers on Earth, can flow slowly over millions of years. Most bizarre of all are a few dark elongated streaks, tens of kilometres across. Initial suggestions that these might be wind-trails, formed of evaporating surface ices dispersed by the atmosphere, did not last long. Triton's atmosphere, inferred from the observation of a stellar occultation, has a pressure of no more than 10 millionths of Earth's, and is mostly nitrogen. There is too little of it to create a trail of sublimed ice.

The explanation settled on a few days after encounter was that the trails were from 'ice volcanoes'. Because nitrogen is just barely frozen at the surface temperature, an overburden of 20 or 30 metres is enough to cause nitrogen ice to liquefy. If fissures develop in the overlying ice, the liquid below will burst out, turn to gas which can shoot several kilometres above the surface, then condense and fall back. The thin surface layer so formed appears dark only in comparison with its surroundings, and may also entrap some of the sublimed dark ice particles in the vicinity.

A similar process may also account for yellow streaks seen on Io, one of Jupiter's moons, where underlying sulphur rather than nitrogen may rush up through surface cracks. Triton would then seem like a jigsaw of pieces from elsewhere in the Solar System, harbouring analogues of the martian polar caps, lunar maria and Io's vulcanism.

\section{Rings, arcs and moons}

BEFORE Voyager got close to Neptune, many astronomers were anticipating confirmation of the existence of many 'ring arcs', or partial rings. But by early this week, only four or five rings had been found, and all were complete, encircling the entire planet. Where did the arcs go?

Observations from Earth of occultations of stars passing behind Neptune suggested partial rings: stars were seen to flicker in brightness as they approached Neptune's disk, but no diminution in brightness was seen as the star emerged at the other side.

Altogether there was evidence for six ring arcs, at various distances from the planet. The early signs from Voyager looked good. JPL scientists announced three weeks before the encounter (see Nature $340,492 ; 1989)$ that they had detected faint reflected light from two partial rings.

But on Tuesday 22 August, new images revealed that the inner arc was in fact a complete ring, and that the other arc was longer than first thought. Much better pictures came down on Saturday, after closest approach, when Voyager could look back to see the ring system shining brightly in forward-scattered rather than reflected sunlight.

The full ring system revealed in these backlit images is complex. The two bright rings, 53,000 and $62,000 \mathrm{~km}$ in radius, are indeed both full rings, but the outer one has conspicuous clumps. Inside the inner bright ring is a faint and rather wide band, $42,000 \mathrm{~km}$ from Neptune and 2,000 km wide. Between the two bright rings is a fainter ring, but rather than a distinct ring this may be the edge of a tenuous, barely visible disk of material extending inwards to the inner bright ring, and possibly beyond.

The final assessment was that groundbased observers had indeed detected clumps in the outer ring - three of the observations gave about the right radius - and made the simple assumption that the ring was incomplete.

But one occultation measurement was both too far out and too strong to be consistent with any observed ring. Despite the astronomical odds, it seems that this occultation was caused by the then unknown moon $1989 \mathrm{~N} 2$, a $200-\mathrm{km}$-wide body happening, at a distance of 500 million $\mathbf{~ k m}$, to pass in front of a background star.

The final haul of moons was six, varying from $42,000 \mathrm{~km}$ out and $50 \mathrm{~km}$ in diameter to $120,000 \mathrm{~km}$ out and $500 \mathrm{~km}$ across. As with the other giant planets, the rings and moons form a complex system, whose properties will take years to puzzle out. Neptune's clumpy outer ring demands explanation, but none is immediately to hand: none of the moons is in the right place or has the right mass to be clearly responsible, by its gravitational influence, for the nonuniformities observed. 